\title{
On Discrete Value Distribution of Certain Compositions
}

\author{
Virginija Garbaliauskiene் $\dot{e}^{a}$, Julija Karaliūnait $\dot{e}^{b}$ and \\ Renata Macaitiené ${ }^{c}$ \\ ${ }^{a}$ Department of Computer Sciences, Šiauliai University \\ Vilniaus str. 141, LT-76353 Šiauliai, Lithuania \\ ${ }^{b}$ Faculty of Fundamental Sciences, Vilnius Gediminas Technical University \\ Sauletekio al. 11, LT-10223 Vilnius, Lithuania \\ ${ }^{c}$ Research Institute, Šiauliai University \\ P. Višinskio str. 25, LT-76351 Šiauliai, Lithuania \\ E-mail(corresp.): virginija.garbaliauskiene@su.lt \\ E-mail: julija.karaliunaite@vgtu.lt \\ E-mail: renata.macaitiene@su.lt
}

Received June 7, 2018; revised October 10, 2018; accepted October 12, 2018

\begin{abstract}
In the paper, we obtain universality theorems and a lower estimate for the number of zeros for the composition $F(\zeta(s, \underline{\alpha} ; \underline{\mathfrak{a}}, \underline{\mathfrak{b}}))$, where $F$ is an operator in the space of analytic functions satisfying the Lipschitz type condition, and $\underline{\zeta}(s, \underline{\alpha} ; \underline{\mathfrak{a}}, \underline{\mathfrak{b}})$ is a collection consisting of periodic and periodic Hurwitz zeta-functions.
\end{abstract}

Keywords: Mergelyan theorem, periodic Hurwitz zeta-function, periodic zeta-function, Rouché theorem, universality.

AMS Subject Classification: 11M41; 41A30.

\section{Introduction}

Let $s=\sigma+i t$ be a complex variable, and $\mathfrak{a}=\left\{a_{m}: m \in \mathbb{N}\right\}$ be a periodic sequence of complex numbers with minimal period $k \in \mathbb{N}$. The periodic zetafunction $\zeta(s ; \mathfrak{a})$ is defined, for $\sigma>1$, by the Dirichlet series

$$
\zeta(s ; \mathfrak{a})=\sum_{m=1}^{\infty} \frac{a_{m}}{m^{s}}
$$

Copyright (c) 2019 The Author(s). Published by VGTU Press

This is an Open Access article distributed under the terms of the Creative Commons Attribution License (http://creativecommons.org/licenses/by/4.0/), which permits unrestricted use, distribution, and reproduction in any medium, provided the original author and source are credited. 
and can be continued meromorphically to the whole complex plane with unique simple pole at the point $s=1$ with residue $a=\frac{1}{k} \sum_{m=1}^{k} a_{m}$. If $a=0$, then $\zeta(s ; \mathfrak{a})$ is an entire function.

Let $\mathfrak{b}=\left\{b_{m}: m \in \mathbb{N}_{0}\right\}, \mathbb{N}_{0}=\mathbb{N} \cup\{0\}$, be one more periodic sequence of complex numbers with minimal period $l \in \mathbb{N}$. The periodic Hurwitz zetafunction $\zeta(s, \alpha ; \mathfrak{b})$ with parameter $\alpha, 0<\alpha \leqslant 1$, is defined, for $\sigma>1$, by the Dirichlet series

$$
\zeta(s, \alpha ; \mathfrak{b})=\sum_{m=0}^{\infty} \frac{b_{m}}{(m+\alpha)^{s}}
$$

and can be continued meromorphically to the whole complex plane with unique simple pole at the point $s=1$ with residue $b=\frac{1}{l} \sum_{m=0}^{l-1} b_{m}$. If $b=0$, then $\zeta(s, \alpha ; \mathfrak{b})$ is an entire function.

This note is devoted to discrete value distribution of collections consisting of periodic and periodic Hurwitz zeta-functions. In [2], the approximation of a collection of analytic functions by discrete shifts of the above collections of zeta-functions has been considered. For $j=1, \ldots, r_{1}$, let $\mathfrak{a}_{j}=\left\{a_{j m}: m \in \mathbb{N}\right\}$ be a periodic sequence of complex numbers with minimal period $q_{j} \in \mathbb{N}$, and $\zeta\left(s ; \mathfrak{a}_{j}\right)$ be the corresponding periodic zeta-function. For $j=1, \ldots, r_{2}$, let $l_{j} \in \mathbb{N}, 0<\alpha_{j} \leqslant 1, b_{j l}=\left\{b_{j l m}: m \in \mathbb{N}_{0}\right\}, l=1, \ldots, l_{j}$, be a periodic sequence of complex numbers with minimal period $q_{j l}$, and let $\zeta\left(s, \alpha_{j} ; \mathfrak{b}_{j l}\right)$ be the corresponding periodic Hurwitz zeta-function. Moreover, let $q$ denote the least common multiple of the periods $q_{1}, \ldots, q_{r_{1}}$, and let $\eta_{1}, \ldots, \eta_{r}$ be the reduced residue system modulo $q$, where $r=\varphi(q)$ is the Euler totient function. Similarly, let $q_{j}$ denote the least common multiple of the periods $q_{1 l_{1}}, \ldots, q_{j l_{j}}$, $j=1, \ldots, r_{2}$. Define the matrices

$$
\begin{aligned}
A & =\left(\begin{array}{cccc}
a_{1 \eta_{1}} & a_{2 \eta_{1}} & \ldots & a_{r_{1} \eta_{1}} \\
a_{1 \eta_{2}} & a_{2 \eta_{2}} & \ldots & a_{r_{1} \eta_{2}} \\
\ldots & \ldots & \ldots & \ldots \\
a_{1 \eta_{r}} & a_{2 \eta_{r}} & \ldots & a_{r_{1} \eta_{r}}
\end{array}\right), \\
B_{j} & =\left(\begin{array}{cccc}
b_{j 10} & b_{j 20} & \ldots & b_{j l_{j} 0} \\
b_{j 11} & b_{j 21} & \ldots & b_{j l_{j} 1} \\
\ldots & \ldots & \ldots & \ldots \\
b_{j 1\left(q_{j}-1\right)} & b_{j 2\left(q_{j}-1\right)} & \ldots & b_{j l_{j}\left(q_{j}-1\right)}
\end{array}\right), \quad j=1, \ldots, r_{2} .
\end{aligned}
$$

For the statement of a joint discrete universality theorem, we use the following notation. Let $D=\left\{s \in \mathbb{C}: \frac{1}{2}<\sigma<1\right\}, \mathcal{K}$ be the class of compact subsets of the strip $D$ with connected complements, $H(K)$ with $K \in \mathcal{K}$ be the class of continuous functions on $K$ that are analytic in the interior of $K$, and let $H_{0}(K)$ be the subclass of $H(K)$ of non-vanishing functions on $K$. Denote by $\mathbb{P}$ the set of all prime numbers, by $\# A$ the cardinality of the set $A$, and define the set

$$
\begin{aligned}
& L\left(\mathbb{P} ; \alpha_{1}, \ldots, \alpha_{r_{2}} ; h, \pi\right) \\
& \quad=\left\{(\log p: p \in \mathbb{P}),\left(\log \left(m+\alpha_{j}\right): m \in \mathbb{N}_{0}, j=1, \ldots, r_{2}\right), \frac{2 \pi}{h}\right\}
\end{aligned}
$$

with $h>0$. Then the main result of [2] is the following theorem. 
Theorem 1. Suppose that the sequences $\mathfrak{a}_{1}, \ldots, \mathfrak{a}_{r_{1}}$ are multiplicative, $\operatorname{rank} A=$ $r_{1}$, the set $L\left(\mathbb{P} ; \alpha_{1}, \ldots, \alpha_{r_{2}} ; h, \pi\right)$ is linearly independent over the field of rational numbers $\mathbb{Q}$, and $\operatorname{rank} B_{j}=l_{j}, j=1, \ldots, r_{2}$. Let $K_{j} \in \mathcal{K}, j=1, \ldots, r_{1}$, $K_{j l} \in \mathcal{K}, j=1, \ldots, r_{2}, l=1, \ldots, l_{j}$, and $f_{j}(s) \in H_{0}\left(K_{j}\right), j=1, \ldots, r_{1}$, $f_{j l}(s) \in H\left(K_{j l}\right), j=1, \ldots, r_{2}, l=1, \ldots, l_{j}$. Then, for every $\varepsilon>0$,

$$
\begin{aligned}
\liminf _{N \rightarrow \infty} \frac{1}{N+1} \#\left\{0 \leqslant k \leqslant N: \sup _{1 \leqslant j \leqslant r_{1}} \sup _{s \in K_{j}}\left|\zeta\left(s+i k h ; \mathfrak{a}_{j}\right)-f_{j}(s)\right|<\varepsilon,\right. \\
\left.\sup _{1 \leqslant j \leqslant r_{2}} \sup _{1 \leqslant j \leqslant l_{j}} \sup _{s \in K_{j l}}\left|\zeta\left(s+i k h, \alpha_{j} ; \mathfrak{b}_{j l}\right)-f_{j l}(s)\right|<\varepsilon\right\}>0 .
\end{aligned}
$$

We note that $N$ runs non-negative integers. Theorem 1 has the following modification.

Theorem 2. Under hypotheses of Theorem 1, the limit

$$
\begin{aligned}
& \lim _{N \rightarrow \infty} \frac{1}{N+1} \#\left\{0 \leqslant k \leqslant N: \sup _{1 \leqslant j \leqslant r_{1}} \sup _{s \in K_{j}}\left|\zeta\left(s+i k h ; \mathfrak{a}_{j}\right)-f_{j}(s)\right|<\varepsilon\right. \\
&\left.\sup _{1 \leqslant j \leqslant r_{2}} \sup _{1 \leqslant j \leqslant l_{j}} \sup _{s \in K_{j l}}\left|\zeta\left(s+i k h, \alpha_{j} ; \mathfrak{b}_{j l}\right)-f_{j l}(s)\right|<\varepsilon\right\}>0
\end{aligned}
$$

exists for all but at most countably many $\varepsilon>0$.

We recall that $D=\left\{s \in \mathbb{C}: \frac{1}{2}<\sigma<1\right\}$. Denote by $H(D)$ the space of analytic functions on $D$ endowed with the topology of uniform convergence on compacta. The aim of this paper is to obtain some analytic properties of the function $F(\underline{\zeta}(s, \underline{\alpha} ; \underline{\mathfrak{a}}, \underline{\mathfrak{b}}))$ for a certain class of operators $F: H^{\kappa}(D) \rightarrow H(D)$, where

$$
\begin{array}{r}
\underline{\zeta}(s, \underline{\alpha} ; \underline{\mathfrak{a}}, \underline{\mathfrak{b}})=\left(\zeta\left(s ; \mathfrak{a}_{1}\right), \ldots, \zeta\left(s ; \mathfrak{a}_{r_{1}}\right), \zeta\left(s, \alpha_{1} ; \mathfrak{b}_{11}\right) \ldots, \zeta\left(s, \alpha_{1} ; \mathfrak{b}_{1 l_{1}}\right), \ldots,\right. \\
\left.\zeta\left(s, \alpha_{r_{2}} ; \mathfrak{b}_{r_{2} 1}\right) \ldots, \zeta\left(s, \alpha_{r_{2}} ; \mathfrak{b}_{r_{2} l_{r_{2}}}\right)\right)
\end{array}
$$

with $\underline{\alpha}=\left(\alpha_{1}, \ldots, \alpha_{r_{1}}\right), \underline{\mathfrak{a}}=\left(\mathfrak{a}_{1}, \ldots, \mathfrak{a}_{r_{1}}\right), \underline{\mathfrak{b}}=\left(\mathfrak{b}_{11}, \ldots, \mathfrak{b}_{1 l_{1}}, \ldots, \mathfrak{b}_{r_{2} 1}, \ldots\right.$, $\left.\mathfrak{b}_{r_{2} l_{r_{2}}}\right)$, and $\kappa=r_{1}+\sum_{j=1}^{r_{2}} l_{j}$.

The space $H(D)$ is metrisable. There exists a sequence of compact sets $\left\{K_{l}: l \in \mathbb{N}\right\} \subset D$ such that $D=\bigcup_{l=1}^{\infty} K_{l}, K_{l} \subset K_{l+1}$ for all $l \in \mathbb{N}$, and if $K \subset D$ is a compact, then $K \subset K_{l}$ for some $l$. Then

$$
\rho\left(g_{1}, g_{2}\right)=\sum_{l=1}^{\infty} 2^{-l} \frac{\sup _{s \in K_{l}}\left|g_{1}(s)-g_{2}(s)\right|}{1+\sup _{s \in K_{l}}\left|g_{1}(s)-g_{2}(s)\right|}, \quad g_{1}, g_{2} \in H(D),
$$

is a metric on $H(D)$ inducing its topology of uniform convergence on compacta. Setting

$$
\underline{\rho}\left(\underline{g}_{1}, \underline{g}_{2}\right)=\max _{1 \leqslant m \leqslant \kappa}\left(\rho\left(g_{1 m}, g_{2 m}\right)\right), \quad \underline{g}_{j}=\left(g_{j 1}, g_{j 2}, \ldots, g_{j \kappa}\right) \subset H^{\kappa}(D), j=1,2,
$$

we obtain the metric which induces the product topology of $H^{\kappa}(D)$.

We note that the sets $K_{l}$ can be chosen with connected complements. For example, we can take closed rectangles. 
Suppose that $\beta_{1}, \ldots, \beta_{\kappa}$ are positive numbers. We say that an operator $F$ : $H^{\kappa}(D) \rightarrow H(D)$ belongs to the class $\operatorname{Lip}\left(\beta_{1}, \ldots, \beta_{\kappa}\right)$ if the following conditions hold:

$1^{\circ}$ For every polynomial $p=p(s)$ and sets $K_{1}, \ldots, K_{r_{1}} \in \mathcal{K}$, there exists

$$
\underline{g}=\left(g_{1}, \ldots, g_{r_{1}}, g_{11}, \ldots, g_{1 l_{1}}, \ldots, g_{r_{2} 1}, \ldots, g_{r_{2} l_{r_{2}}}\right) \in F^{-1}\{p\} \subset H^{\kappa}(D)
$$

such that $g_{j}(s) \neq 0$ on $K_{j}$ for $j=1, \ldots, r_{1}$;

$2^{\circ}$ For all $K \in \mathcal{K}$, there exist a constant $c>0$ and sets $K_{1}, \ldots, K_{\kappa} \in \mathcal{K}$ such that

$$
\begin{gathered}
\sup _{s \in K}\left|F\left(g_{11}(s), \ldots, g_{1 \kappa}(s)\right)-F\left(g_{21}(s), \ldots, g_{2 \kappa}(s)\right)\right| \\
\leqslant c \sup _{1 \leqslant j \leqslant \kappa} \sup _{s \in K_{j}}\left|g_{1 j}(s)-g_{2 j}(s)\right|^{\beta_{j}}
\end{gathered}
$$

for all $\left(g_{j 1}, \ldots, g_{j \kappa}\right) \in H^{\kappa}(D), j=1,2$.

We will prove the following discrete universality theorem on the approximation of analytic functions.

Theorem 3. Suppose that $F \in \operatorname{Lip}\left(\beta_{1}, \ldots, \beta_{\kappa}\right)$, the sequences $\mathfrak{a}_{1}, \ldots, \mathfrak{a}_{r_{1}}$ are multiplicative, $\operatorname{rank} A=r_{1}$, the set $L\left(\mathbb{P} ; \alpha_{1}, \ldots, \alpha_{r_{2}} ; h, \pi\right)$ is linearly independent over $\mathbb{Q}$, and $\operatorname{rank} B_{j}=l_{j}, j=1, \ldots, r_{2}$. Let $K \in \mathcal{K}$ and $f(s) \in H(K)$. Then, for every $\varepsilon>0$,

$$
\liminf _{N \rightarrow \infty} \frac{1}{N+1} \#\left\{0 \leqslant k \leqslant N: \sup _{s \in K}|F(\underline{\zeta}(s, \underline{\alpha} ; \underline{\mathfrak{a}}, \underline{\mathfrak{b}}))-f(s)|<\varepsilon\right\}>0 .
$$

It is not difficult to give an example of $F \in \operatorname{Lip}\left(\beta_{1}, \ldots, \beta_{\kappa}\right)$. Actually, for a given $\left(g_{1}, \ldots, g_{r_{1}}, g_{11}, \ldots, g_{1 l_{1}}, \ldots, g_{r_{2} 1}, \ldots, g_{r_{2} l_{r_{2}}}\right) \in H^{\kappa}(D)$, we take

$$
\begin{gathered}
F\left(g_{1}, \ldots, g_{r_{1}}, g_{11}, \ldots, g_{1 l_{1}}, \ldots, g_{r_{2} 1}, \ldots, g_{r_{2} l_{r_{2}}}\right)=c_{1} g_{1}^{\left(n_{1}\right)}+\cdots+c_{r_{1}} g_{r_{1}}^{\left(n_{r_{1}}\right)} \\
\quad+c_{11} g_{11}^{\left(n_{11}\right)}+\cdots+c_{1 l_{1}} g_{1 l_{1}}^{\left(n_{1 l_{1}}\right)}+\cdots+c_{r_{2} 1} g_{r_{2} 1}^{\left(n_{r_{2} 1}\right)}+\cdots+c_{r_{2} l_{r_{2}}} g_{r_{2} l_{r_{2}}}^{\left(n_{r_{2} l_{r_{2}}}\right)}
\end{gathered}
$$

where $c_{1}, \ldots, c_{r_{1}}, c_{11}, \ldots, c_{1 l_{1}}, \ldots, c_{r_{2} 1}, \ldots, c_{r_{2} l_{r_{2}}} \in \mathbb{C} \backslash\{0\}$ and $n_{1}, \ldots, n_{r_{1}}, n_{11}$, $\ldots, n_{1 l_{1}}, \ldots, n_{r_{2} 1}, \ldots, n_{r_{2} l_{r_{2}}} \in \mathbb{N}$. Then, for every polynomial $p=p(s)$, there exists $\underline{g} \in F^{-1}\{p\}$ such that $g_{j}(s) \neq 0$ on $K_{j}, j=1, \ldots, r_{1}$. Suppose that

$$
p(s)=a_{n} s^{n}+\cdots+a_{0} \quad \text { with } a_{n} \neq 0 .
$$

Then we can take $g=\left(a_{1}, \ldots, a_{r_{1}}, b_{11}, \ldots, b_{1 l_{1}}, \ldots, b_{r_{2} 1}, \ldots, b_{r_{2}\left(l_{r_{2}}-1\right)}, g_{r_{2} l_{r_{2}}}\right)$ with $a_{1}, \ldots, a_{r_{1}} \in \overline{\mathbb{C}} \backslash\{0\}, b_{11}, \ldots, b_{1 l_{1}}, \ldots, b_{r_{2}}, \ldots, b_{r_{2}\left(l_{r_{2}}-1\right)} \in \mathbb{C}$ and

$$
g_{r_{2} l_{r_{2}}}(s)=\frac{1}{c_{r_{2} l_{r_{2}}}}\left(\frac{a_{n} s^{n+n_{r_{2} l_{r_{2}}}}}{(n+1) \cdots\left(n+n_{r_{2} l_{r_{2}}}\right)}+\cdots+\frac{a_{0} s^{n_{r_{2} l_{r_{2}}}}}{1 \cdots n_{r_{2} l_{r_{2}}}}\right) .
$$

This shows that the condition $1^{\circ}$ of the definition of the class $\operatorname{Lip}\left(\beta_{1}, \ldots, \beta_{\kappa}\right)$ is fulfilled. 
For checking the condition $2^{\circ}$ of the class $\operatorname{Lip}\left(\beta_{1}, \ldots, \beta_{\kappa}\right)$, we apply the Cauchy integral formula. Let $K \in \mathcal{K}$, and $K \subset G \subset \hat{K}$ with an open set $G$ and $\hat{K} \in \mathcal{K}$. We take a closed simple contour $L$ lying in $\hat{K} \backslash G$ and enclosing the set $K$. Taking $g_{j 1}, \ldots, g_{j \kappa} \in H^{\kappa}(D), j=1,2$, and using the Cauchy integral formula, we find that, for $s \in K$,

$$
\begin{aligned}
& \left|F\left(g_{11}(s), \ldots, g_{1 \kappa}(s)\right)-F\left(g_{21}(s), \ldots, g_{2 \kappa}(s)\right)\right| \\
& \quad=\left|\sum_{m=1}^{\kappa} c_{m} \frac{n_{m} !}{2 \pi i} \int_{L} \frac{g_{1 m}(z)-g_{2 m}(z)}{(z-s)^{n_{m}+1}} d z\right| \leqslant \sum_{m=1}^{\kappa}\left|c_{m}\right|\left|\hat{c}_{m}\right| \sup _{s \in L}\left|g_{1 m}(s)-g_{2 m}(s)\right| \\
& \quad \leqslant c \sup _{1 \leqslant m \leqslant \kappa} \sup _{s \in \hat{K}}\left|g_{1 m}(s)-g_{2 m}(s)\right|
\end{aligned}
$$

with positive constants $\hat{c}_{m}, m=1, \ldots, \kappa$, and $c$. For simplicity, here we have used the notation $c_{j l} g_{j l}^{\left(n_{j l}\right)}=c_{r_{1}+l_{1}+\cdots+l_{j-1}+l} g_{r_{1}+l_{1}+\cdots+l_{j-1}+l}^{\left(r_{1}+l_{1}+\cdots+l_{j-1}+l\right)}, j=1, \ldots, r_{2}$, $l=1, \ldots, l_{j}$. Thus, by $(1.1)$, we have that the condition $2^{\circ}$ is satisfied with $\beta_{1}=\cdots=\beta_{\kappa}=1$ and $K_{1}=\cdots=K_{\kappa}=\hat{K}$.

Theorem 3, as Theorem 1 , has the following modification.

Theorem 4. Under hypotheses of Theorem 3, the limit

$$
\lim _{N \rightarrow \infty} \frac{1}{N+1} \#\left\{0 \leqslant k \leqslant N: \sup _{s \in K}|F(\underline{\zeta}(s, \underline{\alpha} ; \underline{\mathfrak{a}}, \underline{\mathfrak{b}}))-f(s)|<\varepsilon\right\}>0
$$

exists for all but at most countably many $\varepsilon>0$.

Theorems 1 and 2 are called joint discrete universality theorem for zetafunctions with periodic coefficients. Theorems 3 and 4 are discrete universality theorems for composite functions of zeta-functions with periodic coefficients.

Theorem 3 contains a certain information on zeros of the function $F(\underline{\zeta}(s$, $\underline{\alpha} ; \underline{\mathfrak{a}}, \underline{\mathfrak{b}}))$.

Theorem 5. Suppose that $F \in \operatorname{Lip}\left(\beta_{1}, \ldots, \beta_{\kappa}\right)$, the sequences $\mathfrak{a}_{1}, \ldots, \mathfrak{a}_{r_{1}}$ are multiplicative, $\operatorname{rank} A=r_{1}$, the set $L\left(\mathbb{P} ; \alpha_{1}, \ldots, \alpha_{r_{2}} ; h, \pi\right)$ is linearly independent over $\mathbb{Q}$, and $\operatorname{rank} B_{j}=l_{j}, j=1, \ldots, r_{2}$. Then, for every $\sigma_{1}, \sigma_{2}, \frac{1}{2}<$ $\sigma_{1}<\sigma_{2}<1$, there exists a constant $c=c\left(\sigma_{1}, \sigma_{2}, F, \underline{\alpha}, \underline{\mathfrak{a}}, \underline{\mathfrak{b}}\right)>0$ such that the function $F(\underline{\zeta}(s, \underline{\alpha} ; \underline{\mathfrak{a}}, \underline{\mathfrak{b}}))$, for sufficiently large $N$, has a zero in the disc

$$
\left|s-\left(\sigma_{1}+\sigma_{2}\right) / 2\right| \leqslant\left(\sigma_{2}-\sigma_{1}\right) / 2
$$

for more than $c N$ numbers $k, 0 \leqslant k \leqslant N$.

\section{Proof of universality theorems}

We remind the Mergelyan theorem on the approximation of analytic functions by polynomials [3].

Lemma 1. Let $K \subset \mathbb{C}$ be a compact subset with connected complement, and $f(s)$ be a continuous function on $K$ and analytic in the interior of $K$. Then, for every $\varepsilon>0$, there exists a polynomial $p(s)$ such that

$$
\sup _{s \in K}|f(s)-p(s)|<\varepsilon / 2 .
$$


Next proof of Theorem 3 follows.

Proof. Let $\beta=\min _{1 \leqslant j \leqslant \kappa} \beta_{j}=\min \left(\min _{1 \leqslant j \leqslant r_{1}} \beta_{j}, \min _{1 \leqslant j \leqslant r_{2}} \min _{1 \leqslant j \leqslant l_{j}} \beta_{j l}\right)$. In view of the condition $1^{\circ}$ of the class $\operatorname{Lip}\left(\beta_{1}, \ldots, \beta_{\kappa}\right)$, for the polynomial $p=p(s)$ of Lemma 1 and every $K_{1}, \ldots, K_{r_{1}} \in \mathcal{K}$, there exists an element

$$
\underline{g}=\left(g_{1}, \ldots, g_{r_{1}}, g_{11}, \ldots, g_{1 l_{1}}, \ldots, g_{r_{2} 1}, \ldots, g_{r_{2} l_{r_{2}}}\right) \in F^{-1}\{p\}
$$

such that $g_{j}(s) \neq 0$ on $K_{j}$ for $j=1, \ldots, r_{1}$. Suppose that $c>0$ is from condition $2^{\circ}$ of the class $\operatorname{Lip}\left(\beta_{1}, \ldots, \beta_{\kappa}\right), K_{1}, \ldots, K_{r_{1}}, K_{11}, \ldots, K_{1 l_{1}}, \ldots, K_{r_{2} 1}, \ldots$, $K_{r_{2} l_{r_{2}}}$ correspond the set $K$ in $2^{\circ}$, and that $k \in \mathbb{N}_{0}$ satisfies the inequalities

$$
\begin{aligned}
& \sup _{1 \leqslant j \leqslant r_{1}} \sup _{s \in K_{j}}\left|\zeta\left(s+i k h ; \mathfrak{a}_{j}\right)-g_{j}(s)\right|<c^{-1 / \beta}(\varepsilon / 4)^{1 / \beta}, \\
& \sup _{1 \leqslant j \leqslant r_{2}} \sup _{1 \leqslant l \leqslant l_{j}} \sup _{s \in K_{j l}}\left|\zeta\left(s+i k h, \alpha_{j} ; \mathfrak{b}_{j l}\right)-g_{j l}(s)\right|<c^{-1 / \beta}(\varepsilon / 4)^{1 / \beta} .
\end{aligned}
$$

Then, for $k$ satisfying the above inequalities, we find by $2^{\circ}$ that

$$
\begin{aligned}
& \sup _{s \in K}|F(\underline{\zeta}(s+i k h, \underline{\alpha} ; \underline{\mathfrak{a}}, \underline{\mathfrak{b}}))-p(s)|=\sup _{s \in K}|F(\underline{\zeta}(s+i k h, \underline{\alpha} ; \underline{\mathfrak{a}}, \underline{\mathfrak{b}}))-F(\underline{g})| \\
& \quad \leqslant c \sup _{1 \leqslant j \leqslant r_{1}} \sup _{s \in K_{j}}\left|\zeta\left(s+i k h ; \mathfrak{a}_{j}\right)-g_{j}(s)\right|^{\beta_{j}} \\
& \quad+\sup _{1 \leqslant j \leqslant r_{2}} \sup _{1 \leqslant l \leqslant l_{j}} \sup _{s \in K_{j l}}\left|\zeta\left(s+i k h, \alpha_{j} ; \mathfrak{b}_{j l}\right)-g_{j l}(s)\right|^{\beta_{j l}}<2 c c^{-1} \frac{\varepsilon}{4}=\frac{\varepsilon}{2} .
\end{aligned}
$$

By Theorem 1, the set of $k \in \mathbb{N}_{0}$ satisfying inequalities (2.2) and (2.3) has a positive lower density. Therefore, in view of (2.4),

$$
\liminf _{N \rightarrow \infty} \frac{1}{N+1} \#\left\{0 \leqslant k \leqslant N: \sup _{s \in K}|F(\underline{\zeta}(s+i k h, \underline{\alpha} ; \underline{\mathfrak{a}}, \underline{\mathfrak{b}}))-p(s)|<\frac{\varepsilon}{2}\right\}>0 .
$$

Suppose that $k$ satisfies the inequality

$$
\sup _{s \in K}|F(\underline{\zeta}(s+i k h, \underline{\alpha} ; \underline{\mathfrak{a}}, \underline{\mathfrak{b}}))-p(s)|<\frac{\varepsilon}{2} .
$$

Then, taking into account inequality (2.1), we have for such $k$

$$
\begin{aligned}
\sup _{s \in K} & |F(\underline{\zeta}(s+i k h, \underline{\alpha} ; \underline{\mathfrak{a}}, \underline{\mathfrak{b}}))-f(s)| \\
& \leqslant \sup _{s \in K}|F(\underline{\zeta}(s+i k h, \underline{\alpha} ; \underline{\mathfrak{a}}, \underline{\mathfrak{b}}))-p(s)|+\sup _{s \in K}|f(s)-p(s)|<\varepsilon .
\end{aligned}
$$

Therefore,

$$
\begin{aligned}
\{0 & \left.\leqslant k \leqslant N: \sup _{s \in K}|F(\underline{\zeta}(s+i k h, \underline{\alpha} ; \underline{\mathfrak{a}}, \underline{\mathfrak{b}}))-p(s)|<\frac{\varepsilon}{2}\right\} \\
& \subset\left\{0 \leqslant k \leqslant N: \sup _{s \in K}|F(\underline{\zeta}(s+i k h, \underline{\alpha} ; \underline{\mathfrak{a}}, \underline{\mathfrak{b}}))-f(s)|<\varepsilon\right\},
\end{aligned}
$$


and the theorem follows by (2.5).

Unfortunately, Theorem 4 does not follows directly from Theorem 2, therefore, we will give its direct proof.

Denote by $\mathcal{B}(X)$ the Borel $\sigma$-field of the space $X$, let $S=\{g \in H(D)$ : $g(s) \neq 0$ or $g(s) \equiv 0\}$, and, for $A \in \mathcal{B}(H(D))$, define

$$
P_{N}(A)=\frac{1}{N+1} \#\{1 \leqslant k \leqslant N: \underline{\zeta}(s+i k h, \underline{\alpha} ; \underline{\mathfrak{a}}, \underline{\mathfrak{b}}) \in A\} .
$$

Lemma 2. Suppose that the sequences $\mathfrak{a}_{1}, \ldots, \mathfrak{a}_{r_{1}}$ are multiplicative, $\operatorname{rank} A=$ $r_{1}$, the set $L\left(\mathbb{P} ; \alpha_{1}, \ldots, \alpha_{r_{2}} ; h, \pi\right)$ is linearly independent over $\mathbb{Q}$, and $\operatorname{rank} B_{j}=$ $l_{j}, j=1, \ldots, r_{2}$. Then $P_{N}$, as $N \rightarrow \infty$, converges weakly to a certain probability measure $P_{\underline{\zeta}}$ with support $S^{r_{1}} \times H(D)^{\kappa-r_{1}}$.

The lemma is Proposition 3.1 of [2].

Lemma 3. Suppose that $F \in \operatorname{Lip}\left(\beta_{1}, \ldots, \beta_{\kappa}\right)$. Then

$$
P_{N, F}(A) \stackrel{\text { def }}{=} \frac{1}{N+1} \#\{0 \leqslant k \leqslant N: F(\underline{\zeta}(s+i k h, \underline{\alpha} ; \underline{\mathfrak{a}}, \underline{\mathfrak{b}})) \in A\}, A \in \mathcal{B}(H(D)),
$$

converges weakly to $P_{\underline{\zeta}} F^{-1}$ as $N \rightarrow \infty$. Moreover, the support of $P_{\underline{\zeta}} F^{-1}$ is the whole of $H(D)$.

Proof. We recall that $P_{\zeta} F^{-1}(A)=P_{\zeta}\left(F^{-1} A\right)$ for $A \in \mathcal{B}(H(D))$. The condition $2^{\circ}$ of the class $\operatorname{Lip}\left(\bar{\beta}_{1}, \ldots, \beta_{\kappa}\right)$ shows that the operator $F$ is continuous. Moreover, by the definitions of $P_{N}$ and $P_{N, F}$, we have that $P_{N, F}=P_{N} F^{-1}$. Therefore, Lemma 2, Theorem 5.1 of [1] and the continuity of $F$ prove the weak convergence of $P_{N, F}$ to $P_{\underline{\zeta}} F^{-1}$ as $N \rightarrow \infty$.

The condition $1^{\circ}$ of the class $\operatorname{Lip}\left(\beta_{1}, \ldots, \beta_{\kappa}\right)$ implies that, for each polynomial $p=p(s)$, there exists

$$
\begin{array}{r}
\underline{g}=\left(g_{1}, \ldots, g_{r_{1}}, g_{11}, \ldots, g_{1 l_{1}}, \ldots, g_{r_{2} 1}, \ldots, g_{r_{2} l_{r_{2}}}\right) \\
\in\left(F^{-1}\{p\}\right) \cap\left(S^{r_{1}} \times H^{\kappa-r_{1}}(D)\right) .
\end{array}
$$

Actually, if $g_{j}(s) \neq 0$ on every $K_{j} \in \mathcal{K}, j=1, \ldots, r_{1}$, then $g_{j} \in S, j=1, \ldots, r_{1}$, because if $g_{j}(s)=0$ on $D$, then in view of the equality $D=\bigcup_{l=1}^{\infty} \hat{K}_{l}$ with $\hat{K}_{l} \in \mathcal{K}$ from the definition of the metric $\rho$, we obtain $g_{j}\left(\hat{K}_{l}\right)=0$ for some $l$.

We take an arbitrary $g \in H(D)$ and its open neighbourhood $G$. Then, by the continuity of $F$, the set $F^{-1} G$ is open as well. In virtue of Lemma 2 , there exists a polynomial $p=p(s)$ lying in $G$. Therefore, $F^{-1}\{p\} \subset F^{-1} G$, and by the above remark, the set $F^{-1} G$ contains an element of $S^{r_{1}} \times H^{\kappa-r_{1}}(D)$. Hence, Lemma 2 implies the inequality $P_{\underline{\zeta}}\left(F^{-1} G\right)>0$. Thus,

$$
P_{\underline{\zeta}} F^{-1}(G)=P_{\underline{\zeta}}\left(F^{-1} G\right)>0 \text {. }
$$

Since $g$ and $G$ are arbitrary, this shows that the support of the measure $P_{\underline{\zeta}} F^{-1}$ is the whole of $H(D)$. 
Next we give the proof of Theorem 4 .

Proof. Let the polynomial $p(s)$ satisfy (2.1). Define the set

$$
G_{\varepsilon}=\left\{g \in H(D): \sup _{s \in K}|g(s)-p(s)|<\varepsilon / 2\right\} .
$$

By the second part of Lemma 3 , the set $G_{\varepsilon}$ is an open neighbourhood of the element of the support of the measure $P_{\underline{\zeta}} F^{-1}$. Thus, $P_{\underline{\zeta}} F^{-1}\left(G_{\varepsilon}\right)>0$. We recall that the set $A \in \mathcal{B}(H(D))$ is a continuity set of the measure $P_{\zeta} F^{-1}$ if $P_{\underline{\zeta}} F^{-1}(\partial A)=0$, where $\partial A$ is a boundary of the set $A$. Define one more set

$$
\hat{G}_{\varepsilon}=\left\{g \in H(D): \sup _{s \in K}|g(s)-f(s)|<\varepsilon\right\} .
$$

Then the boundary $\partial \hat{G}_{\varepsilon}$ lies in the set

$$
\left\{g \in H(D): \sup _{s \in K}|g(s)-f(s)|=\varepsilon\right\},
$$

therefore, $\partial \hat{G}_{\varepsilon_{1}} \cap \partial \hat{G}_{\varepsilon_{2}}=\varnothing$ for distinct positive $\varepsilon_{1}$ and $\varepsilon_{2}$. This shows that the set $\hat{G}_{\varepsilon}$ is a continuity set of $P_{\zeta} F^{-1}$ for all but at most countably many $\varepsilon>0$. Moreover, the definitions of $G_{\varepsilon}$ and $\hat{G}_{\varepsilon}$ together with (2.1) imply the inclusion $G_{\varepsilon} \subset \hat{G}_{\varepsilon}$. Hence,

$$
P_{\underline{\zeta}} F^{-1}\left(\hat{G}_{\varepsilon}\right) \geqslant P_{\underline{\zeta}} F^{-1}\left(G_{\varepsilon}\right)>0 \text {. }
$$

Using the equivalent of weak convergence of probability measures in terms of continuity sets, by the first part of Lemma 3 and (2.6), we obtain that

$$
\lim _{N \rightarrow \infty} P_{N, F}\left(\hat{G}_{\varepsilon}\right)=P_{\underline{\zeta}} F^{-1}\left(\hat{G}_{\varepsilon}\right)>0
$$

for all but at most countably many $\varepsilon>0$. This and the definitions of $P_{N, F}$ and $\hat{G}_{\varepsilon}$ prove the theorem.

\section{Proof of Theorem 5}

For convenience, we remind the Rouché theorem.

Lemma 4. Suppose that $G$ is a domain in $\mathbb{C}, K$ is a compact subset of $G$, and $f(s)$ and $g(s)$ are analytic functions on $G$ such that

$$
|f(s)-g(s)|<|f(s)|
$$

for every point $s$ in the boundary of $K$. Then $f(s)$ and $g(s)$ have the same number of zeros in the interior of $K$, taking into account multiplicities. 
Proof of the lemma can be found, for example, in [4]. Now, we prove Theorem 5.

Proof. Let, for brevity,

$$
\sigma_{0}=\frac{\sigma_{1}+\sigma_{2}}{2} \text { and } \quad r_{0}=\frac{\sigma_{2}-\sigma_{1}}{2}
$$

We take $f(s)=s-\sigma_{0}$ in Theorem 3. Then, by the latter theorem, for every $\varepsilon>0$, the set of $k \in \mathbb{N}_{0}$ satisfying the inequality

$$
\sup _{\left|s-\sigma_{0}\right| \leqslant r_{0}}\left|F(\underline{\zeta}(s+i k h, \underline{\alpha} ; \underline{\mathfrak{a}}, \underline{\mathfrak{b}}))-\left(s-\sigma_{0}\right)\right|<\varepsilon
$$

has a positive lower density. We choose $\varepsilon$ to satisfy

$$
0<\varepsilon<\frac{1}{20} \inf _{\left|s-\sigma_{0}\right|=r_{0}}\left|s-\sigma_{0}\right|=\frac{r_{0}}{20}
$$

Then we have that the functions $F(\zeta(s+i k h, \underline{\alpha} ; \underline{\mathfrak{a}}, \underline{\mathfrak{b}}))$ and $s-\sigma_{0}$ on the disc $\left|s-\sigma_{0}\right| \leqslant r_{0}$ satisfy the conditions of Lemma 4. Since, obviously, the function $s-\sigma_{0}$ has one zero in the disc $\left|s-\sigma_{0}\right|<r_{0}$, we find that also the function $F(\underline{\zeta}(s+i k h, \underline{\alpha} ; \underline{\mathfrak{a}}, \underline{\mathfrak{b}}))$ has only one zero in that disc. However, the number of $k$ satisfying inequality (3.1), for sufficiently large $N$, is greater than $c N$ with a certain constant $c>0$ depending on $\sigma_{1}, \sigma_{2}, F, \underline{\alpha}$, and $\underline{\mathfrak{a}}, \underline{\mathfrak{b}}$. The theorem is proved.

\section{References}

[1] P. Billingsley. Convergence of Probability Measures. Willey, New York, 1968.

[2] A. Laurinčikas. Joint discrete universality for periodic zeta-functions. Quaest. Math., 2018. https://doi.org/10.2989/16073606.2018.1481891.

[3] S.N. Mergelyan. Uniform approximations to functions of complex variable. Usp. Mat. Nauk., 7:31-122, 1952 (in Russian).

[4] D. Sarason. Complex Function Theory. Amer. Math. Soc., 2007. 